1.6 to $5 \mathrm{~mm}$. Twenty-nine patients were given non-selective ventilation and twenty-three were anesthetized using selective right-lung ventilation using a $2-\mathrm{F}$ balloon catheter for arterial embolectomy. A left lateral mini-thoracotomy was performed in all infants and PDA closure achieved by double ligation using zero silk sutures.

Results The median operative time and mean length of hospital stay were 45 minutes and 90 days, respectively. No major hemorrhage requiring blood transfusion occurred during the surgery. The survival rate until hospital discharge was $88.5 \%$. There were no mortalities associated with the surgery itself. Six (11.5\%) neonates died postoperatively because of prematurity $(\mathrm{P}$-value $=1.000)$. Pneumonia and atelectasis were among the few complications encountered post ligation. An association was recognized between ventilation and surgical complications; that is neonates who underwent selective right ventilation did not experience any of the complications mentioned above in comparison to those who were put under non-selective ventilation (P-value $<0.001$ ).

Conclusions Closure of PDA by double ligation via a left mini-thoracotomy in small premature infants proved to be safe and effective in providing pediatric surgeons adequate exposure within confined and delicate anatomic spaces. No procedure related mortalities or major complications were encountered. It is a useful option in neonates with failed medical management of PDA especially in preemies where performing VATS is challenging.

\section{TO COMPARE EFFECTIVENESS OF INFRAREDSPECTROSCOPIC TECHNOLOGY WITH CONVENTIONAL PERIPHERAL VENOUS ACCESS IN CHILDREN: RANDOMIZED CONTROL STUDY}

Borra Ranganath, Raj Dhar Dutt, Yogendra Singh Varma, Ajay Gaur. India

\subsection{6/bmjpo-2021-RCPCH.73}

Background Although considered to be a routine minor intervention, intravenous cannulation can often be difficult and potentially painful. The implications maybe more pronounced in younger children. Otica pediatric LED uses near infra-red light to enhance the visibility of veins and thus to negotiate difficult venous access. In our study we hoped to explore the utility of NEAR INFRARED TECHNOLOGY in children with difficult peripheral venous access.

Objectives To assess the superiority of near infra-red spectroscopic technology over conventional vein viewing techniques in children with difficult peripheral venous access.

Methods From November 2018 to August 2020, 509 children aged 3 to 36 months satisfying the inclusion criteria for difficult cannulation (based on DIVA score) were included. Randomization was attempted by employing Otica pediatric LED (intervention group) and conventional methods (control group) every alternate fortnightly by the same set of residents. The median no. of pricks before successful cannulation per child in each group, and the proportion of first attempt success rate in each group were the primary end points.

Results The median no. of attempts was lower in the intervention group (1; IQR 1-2) than in the control group (2; IQR $1-3, \mathrm{p}<0.001)$. Proportion of cannulations successful in the first attempt was 64\% (136 of 211) in the intervention group and 40\% (119 out of 298$)$ in the control group ( $p<0.001)$. From the sub-group analysis we found significant difference
Abstract 132 Table 1 Proportion of success at first attempt cannulation (Age wise)

\begin{tabular}{llll}
\hline Age & Conventional & Intervention & $\begin{array}{l}\text { Chi square } \\
\text { P value }\end{array}$ \\
\hline 3 months to 6 months & $41 / 115(36 \%)$ & $56 / 84(67 \%)$ & $0.001^{*}$ \\
7 months to 12 months & $29 / 83(35 \%)$ & $32 / 52(63 \%)$ & $0.001^{*}$ \\
13 months to 18 months & $27 / 53(51 \%)$ & $26 / 40(65 \%)$ & 0.175 \\
19 months to 24 months & $6 / 10(60 \%)$ & $7 / 11(64 \%)$ & 0.864 \\
25 months to 30 months & $8 / 16(50 \%)$ & $4 / 7(58 \%)$ & 0.752 \\
31 months to 36 months & $8 / 21(38 \%)$ & $10 / 16(63 \%)$ & 0.141 \\
\hline
\end{tabular}

Abstract 132 Table 2 Median number of pricks required to successfully cannulate (Nutrition wise)

\begin{tabular}{llllll}
\hline Weight/height & Conventional & & Intervention & P value \\
\hline & Median (IQR) & N & Median (IQR) & N & \\
\hline & $2(1-3)$ & $48 / 102$ & $1(1-2)$ & $45 / 65$ & $0.002^{*}$ \\
\hline $\begin{array}{l}\text { Sev Z SCORE } \\
\text { Seve acute }\end{array}$ & & & & & \\
Malnutrition & & & & & \\
-3 TO -2 Z Moderate & $2(1-3)$ & $26 / 56$ & $1(1-2)$ & $26 / 42$ & 0.112 \\
Acute Malnutrition & & & & & \\
-2 TO -1 Z SCORE & $2(1-3)$ & $19 / 50$ & $1(1-2)$ & $22 / 33$ & 0.170 \\
-1 TO MEDIAN & $2(1-3)$ & $18 / 51$ & $1(1-2)$ & $26 / 33$ & $0.01^{*}$ \\
$>$ MEDIAN & $2(1-2)$ & $08 / 39$ & $2(1-3)$ & $17 / 37$ & $0.027^{*}$ \\
\hline
\end{tabular}

between both the groups when analysed across the age-groups as well as the nutritional status. An interesting finding in the trial is that the advantage with the NIR device was significant in children requiring more than one instance of cannulation (those that are not IV line naive).

Conclusions In our study we found a significant clinical advantage with the use of Otica pediatric LED consistent across the sub groups. However to vouch for any routine recommendation will require a larger and perhaps a multicenter study to begin with. While the busy and shuffling schedule of a residency inherently weighs down on any interventional study, the results of our study calls for an optimistic investment into the research in near infrared technology for standard cannulation procedures.

\section{REDUCING BLOOD TESTING IN PICU, A QUICK QI PROJECT}

Fadi Maghrabia, Jo Lumsden, Santosh Sundararajan. UK

\subsection{6/bmjpo-2021-RCPCH.74}

\section{Background}

Introduction Critically ill children are at significant risk of repeated blood sampling for laboratory testing, which could lead to nosocomial anaemia and blood transfusions ${ }^{1}$. We aimed to optimise blood testing and the associated costs in PICU without adversely impacting patient safety and outcome. Objectives As part of the quality improvement initiative, a bedside guideline for common blood tests was introduced in 2014 based on a patient stratification system. We wanted to check if our practice is compliant with the guideline. 